\title{
A Low-Power Multicarrier-CDMA Downlink Baseband Receiver for Future Cellular Communication Systems
}

\author{
Pei-Yun Tsai, Member, IEEE, and Tzi-Dar Chiueh, Senior Member, IEEE
}

\begin{abstract}
In this paper, design and implementation of a baseband receiver integrated circuit (IC) for a downlink multi-carrier code-division multiple access (MC-CDMA) system are presented. This MC-CDMA system aims to provide higher data transmission capacity than the current wide-band CDMA systems in mobile cellular communication environments. The proposed chip provides a robust tracking mechanism for synchronization errors and an accurate channel estimation strategy to overcome the challenge of outdoor fast-fading channels. Besides, low-power and lowcomplexity architecture design techniques are adopted to satisfy mobile receiver needs. Experimental results of the designed baseband receiver integrated circuit demonstrate its superior system performance and great reduction in power consumption. The chip was fabricated in a $0.18-\mu \mathrm{m} \mathrm{CMOS} \mathrm{technology} \mathrm{with} \mathrm{a} \mathrm{core} \mathrm{area} \mathrm{of}$ $2.6 \mathrm{~mm} \times 2.6 \mathrm{~mm}$. It can support up to 21.7-Mbps uncoded data rate in a 5-MHz bandwidth. When running at 5.76 MHz, its power consumption is as low as $9.9 \mathrm{~mW}$ from a supply voltage of $1.1 \mathrm{~V}$.
\end{abstract}

Index Terms-Baseband receiver, code-division multiple access (CDMA), multi-carrier modulation.

\section{INTRODUCTION}

D IRECT-SEQUENCE spread-spectrum (DSSS) codedivision multiple access (CDMA) is a multiple access scheme adopted in the third generation (3G) cellular communication standard to provide high capacity and high transmission rate over conventional multiple access methods such as frequency-division multiple access (FDMA) and time-division multiple access (TDMA). However, it is also well known that frequency-selective multi-path fading inevitably encountered in broadband wireless channels can severely degrade DSSS CDMA system performance. Although coherent Rake combining can exploit path diversity and enhance receiver performance, a large number of paths to be resolved in broadband systems entail high receiver complexity. What is worse, multiple access interference (MAI) caused by channel dispersion can not be effectively suppressed using Rake combining.

Manuscript received May 19, 2006; revised February 6, 2007. This work was supported in part by the National Science Council, Taiwan, R.O.C. under Grant NSC94-2219-E-002-027 and Grant NSC95-2219-E-002-020. This paper was recommended by Associate Editor F. C. M. Lau.

P.-Y. Tsai was with the Graduate Institute of Electronics Engineering and the Department of Electrical Engineering, National Taiwan University, Taipei, Taiwan 10617, R.O.C. She is now with the Department of Electrical Engineering, National Central University, Jhong-Li, Taiwan 32001, R.O.C.

T.-D. Chiueh is with the Graduate Institute of Electronics Engineering and the Department of Electrical Engineering, National Taiwan University, Taipei, Taiwan 10617, R.O.C. (e-mail: chiueh@cc.ee.ntu.edu.tw).

Digital Object Identifier 10.1109/TCSI.2007.904593
Recently, a new multi-carrier modulation scheme, orthogonal frequency-division multiplexing (OFDM) attracts much attention from the communications research community. In an OFDM communication system, a frequency-selective-faded wide-band signal is partitioned into a large number of flat-faded narrowband signals. The adverse channel effects can consequently be mitigated by using a simple one-tap frequency-domain equalizer (FEQ). The longer symbol duration in OFDM systems also makes their signals more resistant to time dispersion caused by the channel. With the guard interval between every pair of symbols, inter-symbol interference (ISI) can be contained and eliminated. In addition to the above advantages, the mutually overlapped spectra of the subcarriers provide efficient utilization of the allocated spectrum given that cross-symbol discontinuities are smoothed by time-domain windowing, filtering, or a combination of both.

In light of these advantages, OFDM has been adopted in several standards, such as digital video broadcasting-terrestrial (DVB-T), digital subscriber line (DSL), and IEEE 802.11a/g wireless LAN. In the third generation partnership project (3GPP), a feasibility study of OFDM for universal mobile telecommunications system terrestrial radio access network (UTRAN) enhancement is conducted to evaluate its potential benefits and has taken into consideration the possibility of incorporating the OFDM technique [1]. As such, multi-carrier CDMA (MC-CDMA), which combines OFDM and CDMA techniques, has been proposed in [2]-[4]. Inheriting advantages from both multi-carrier modulation and CDMA, MC-CDMA is capable of combating frequency-selective fading channels as well as offering high data rate transmission in a multi-user environment. Moreover, the adopted orthogonal variable spreading factor (OVSF) codes spread the original data symbol over several subcarriers, making frequency diversity reception multi-rate transmission possible. In light of all the above advantages, MC-CDMA has become one of the attractive candidates for next-generation mobile communications. Field trials of MC-CDMA systems have been conducted in Japan and several ongoing projects regarding MC-CDMA standardization in future broadband mobile systems are under way in Europe [3], [4].

Although wireless communication solutions using OFDM are mature and plentiful [5], [6], applying OFDM in the mobile cellular communication environment can be quite different. In the cellular environment, time-varying fast-fading channels caused by high mobility pose a severe challenge to synchronization and channel estimation tasks in the MC-CDMA receiver. A robust 
tracking mechanism for the synchronization parameters must be established since multi-carrier modulation is notorious for its vulnerability to synchronization errors. In fast-fading mobile cellular environments, the MC-CDMA receiver must have an agile and precise channel estimation algorithm to follow variation in channel responses. In addition, owing to larger cell size and thus longer delay spread, the MC-CDMA system must use a smaller subcarrier bandwidth. This entails more subcarriers in OFDM modulation and the receiver complexity will be much higher.

In this paper, a downlink baseband receiver architecture using MC-CDMA suitable for mobile cellular communication in urban areas is proposed. The MC-CDMA receiver can support up to 21.7-Mbps (uncoded) downlink data transmission and has compatible carrier frequency and bandwidth with the current 3G wide-band CDMA (W-CDMA) standard. The receiver IC dissipates only $9.9 \mathrm{~mW}$ at $5.76-\mathrm{MHz}$ operating frequency from a supply voltage of $1.1 \mathrm{~V}$. Features of the proposed receiver include the following:

- provision of a robust and precise tracking mechanism for carrier frequency offset (CFO) and sampling clock offset (SCO).

- provision of a smart-channel estimation mechanism according to Doppler frequency;

- adoption of a specialized channel interpolation algorithm with increased accuracy of channel estimation in fastfading channels.

Two circuit design techniques are also adopted in the IC, including:

- implementation of a custom-designed bit-reversal architecture with reduced SRAM requirements;

- implementation of a low-complexity high-performance equalizer architecture.

The paper is organized as follows. Description as well as parameters of the MC-CDMA system are first introduced in Section II. Then, the baseband transceiver function blocks are presented in Section III. Section IV describes the baseband transceiver architecture and several implementation issues crucial to achieving low power and small area. In Section V, the physical design of the proposed chip and its measurement results are given. Finally Section VI concludes this paper.

\section{SYSTEM DESCRIPTION}

In Table I, we summarize several important parameters of the proposed MC-CDMA system [7], [8]. The proposed system uses the same RF frequency and signal bandwidth as those of the $3 \mathrm{G}$ standards, i.e., $2 \mathrm{GHz}$ and $5 \mathrm{MHz}$, since its major goal is to increase the downlink data rate of the current 3G W-CDMA cellular communication system in urban areas. Moreover, we reserve $5 \%$ bandwidth on both ends of the signal band as guard bands. To facilitate future dual-mode receiver design, the ADC sampling rate is set to $5.76 \mathrm{MHz}, 1.5$ times the chip rate in the W-CDMA system (3.84 MHz).

The channel models provided by 3GPP [9] specify that the maximum excess delays are $2.14 \mu$ s and over $10 \mu$ s in typical urban and bad urban areas, respectively, and the highest mobility supported is up to $120 \mathrm{~km} / \mathrm{h}$. Accordingly, we set the guard interval to be longer than $10 \mu \mathrm{s}$. Also, the maximum Doppler
TABLE I

MC-CDMA SYSTEM PARAMETERS

\begin{tabular}{|l|c|}
\hline RF Frequency & $2 \mathrm{GHz}$ \\
\hline Signal Bandwidth & $5 \mathrm{MHz}$ \\
\hline Sampling Frequency & $5.76 \mathrm{MHz}$ \\
\hline FFT Size (N) & 1024 \\
\hline Subcarrier Spacing & $5.625 \mathrm{KHz}$ \\
\hline Guard Interval & $11.1 \mu \mathrm{s}$ \\
\hline Symbol Time & $188.9 \mu \mathrm{s}$ \\
\hline Number of Data Subcarriers & 768 \\
\hline Number of Pilot Subcarriers & 33 \\
\hline Maximum Data Rate (Uncoded) & $21.7 \mathrm{Mbps}$ \\
\hline
\end{tabular}

frequency, $222 \mathrm{~Hz}$, limits the OFDM symbol duration to be shorter than $200 \mu \mathrm{s}$, such that the signal-to-interference ratio can be made higher than $25 \mathrm{~dB}$ [10], [11]. Given the sampling rate and the symbol duration, the fast Fourier transform (FFT) size is set to 1024 . Out of the 1024 subcarriers, 768 subcarriers are used to transmit data and 33 pilot subcarriers are uniformly inserted for synchronization/channel estimation. Three signal constellations, namely, quadrature phase-shift keying (QPSK), 16 quadrature ampliture modulation (QAM), and 64 QAM can be used and the maximum uncoded data rate reaches $21.7 \mathrm{Mbps}$. The orthogonal variable spreading factor (OVSF) codes [12] spread the user data onto a number of subcarriers. The same FFT size, guard interval ratio, and guard band ratio are adopted in the feasibility study of the 3GPP UTRAN OFDM enhancement [1].

\section{TRANSMITTER AND RECEIVER DESIGN}

\section{A. Transmitter}

The transmitter block diagram is shown in Fig. 1(a). Data from each user first pass through a constellation mapper, get spread by respective OVSF code, and then are combined with signals from other users. Since the length of the OVSF code may be less than 768, thus one may transmit several pieces of data in one MC-CDMA symbol. To achieve maximum frequency diversity, we spread the data onto subcarriers that are as far apart as possible. In other words, adjacent subcarriers are assigned to different pieces of data that have been spread by the same chip of the OVSF code [13].

Comb-type uniformly distributed pilot subcarriers are inserted to facilitate synchronization and channel estimation tasks in the receiver. An OFDM modulator then transforms the frequency-domain signals to time-domain MC-CDMA signals. Afterwards, training symbols are transmitted periodically in the time domain so as to ensure continuous and reliable communication links. The allocation of pilot subcarriers and training symbols is depicted in Fig. 1(b). The training symbols play an important role when a receiver starts up and they also provide for frequency-domain channel estimation in stationary and quasi-stationary channels. A training symbol consists of two identical halves [14], making all odd-numbered subcarriers zero. With such an arrangement, the receiver can achieve more robust symbol boundary detection than the design that uses only the cyclic prefix. Moreover, the even-numbered 


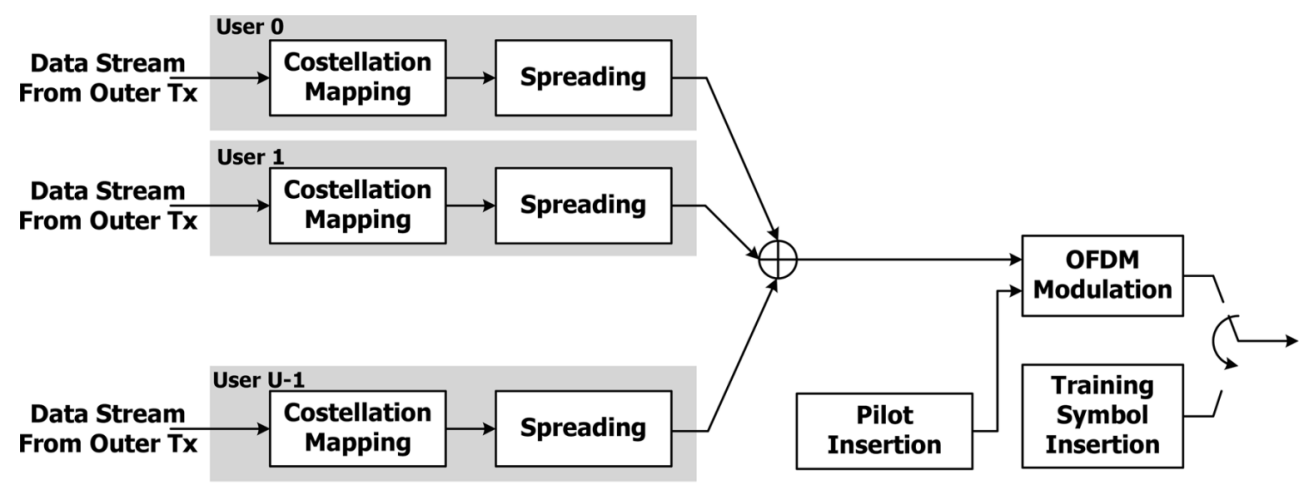

(a)

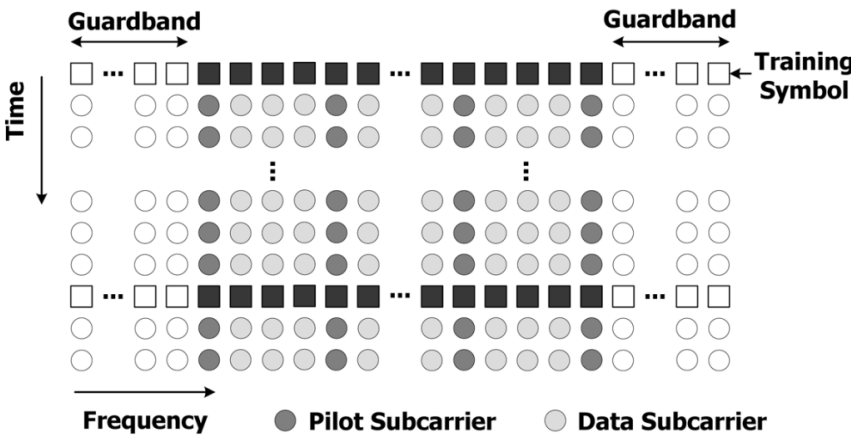

(b)

Fig. 1. (a) Block diagram of the MC-CDMA transmitter. (b) Allocation of pilot subcarriers and training symbols.

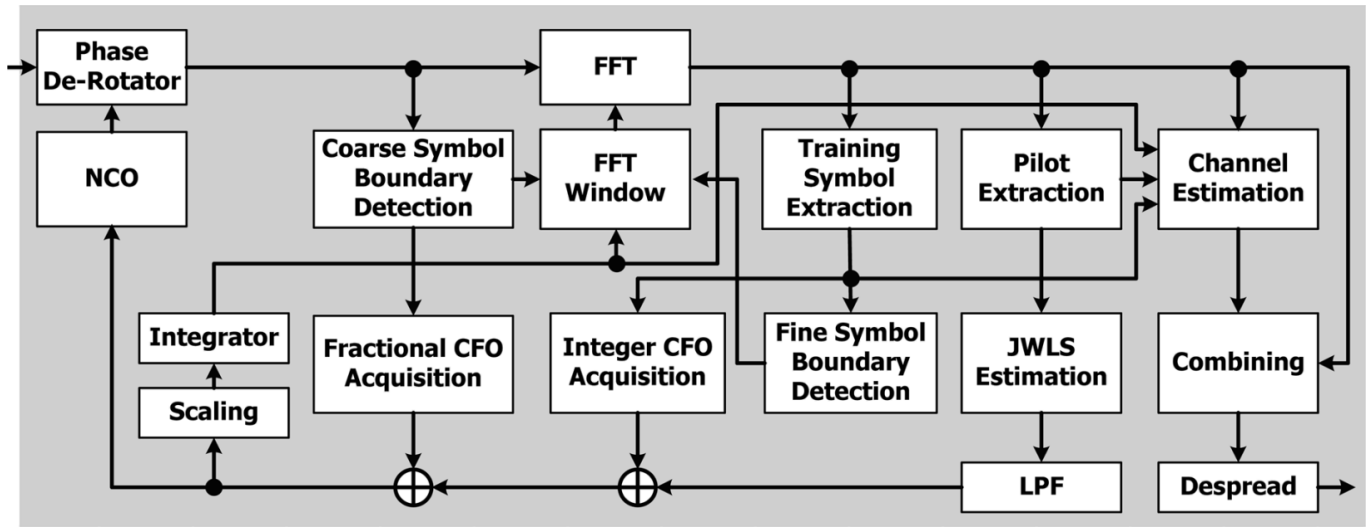

Fig. 2. Block diagram of the MC-CDMA receiver.

subcarriers in the training symbol are differentially encoded with a pseudonoise ( $\mathrm{PN}$ ) sequence to expedite integer CFO acquisition [15].

\section{B. Receiver}

Fig. 2 depicts the block diagram of the baseband receiver for the proposed downlink MC-CDMA communication system. In the receiver, a symbol timing detector, a carrier recovery loop, and a sampling clock recovery loop are integrated to deal with the synchronization issues. Owing to the precise synchronization requirements in the multi-carrier systems, rough acquisition which speeds up the loop convergence and subsequent tracking mechanism which enhances the accuracy are both implemented [7].
When the receiver starts up and after the automatic gain control circuit has settled, coarse symbol timing is detected by looking for a training symbol. The receiver adopts a delay correlator with a delay of $N / 2$, where $N$ is the FFT size. The output of the delay correlation is then passed to a moving average block, whose output peak indicates the end of a training symbol [7]. Assume that the normalized CFO to the subcarrier spacing is given by $\epsilon$. Then, the phase shift between the two identical halves of the training symbol is $\pi \epsilon$. We divide $\epsilon$ into two components: $\epsilon_{I}$ and $\epsilon_{f}$, where $\epsilon_{I}$ is the nearest even integer to $\epsilon$ and $\epsilon=\epsilon_{I}+\epsilon_{f},-1 \leq \epsilon_{f}<1$. Then the maximum likelihood estimation of the fractional CFO, $\hat{\epsilon}_{f}$, can be derived simultaneously when the symbol timing is decided.

Integer CFO causes signal displacement in the frequency domain. The data on the even-numbered frequency-domain 
subcarriers of the training symbol are differentially encoded by a PN sequence $\left(c_{k}\right)$. Due to the excellent autocorrelation property of the PN sequence, the integer $\mathrm{CFO}$ can be obtained by finding the maximum in the auto-correlation output $\left|\sum_{k \in \mathbf{K}} Z_{2 k+g} Z_{2 k+g+2}^{*} c_{k}^{*}\right|$, where $Z_{k}$ is the received frequency-domain signal of the training symbol and $\mathbf{K}$ is a set of subcarriers that have been differentially encoded by the PN sequence [15]. The integer CFO $\left(\epsilon_{I}\right)$ is the index $(g)$ that corresponds to the maximum output.

After integer CFO acquisition, the receiver activates fine symbol timing detection and adjusts the symbol boundary to an ISI-free position. Because the degree of phase shift between adjacent subcarrier data in the frequency domain reflects the corresponding symbol timing offset in the time domain, the receiver gets an estimate of the offset in symbol boundary by computing the phase shift between signals in neighboring subcarriers and then averaging the phase shifts over all subcarriers in the training symbol. Note that since similar computation is required in estimating the integer $\mathrm{CFO}$ and the symbol timing offset, these two blocks share one complex multiplier.

MC-CDMA signal, as OFDM signal, is susceptible to synchronization errors which corrupt the orthogonality among subcarriers. In such unfortunate cases, both inter-carrier interference (ICI) and MAI will be present [16]. It is thus imperative that MC-CDMA receivers adopt accurate and robust residual synchronization error estimation algorithms to deal with inaccurate initial CFO estimation and non-stationary channels so that ICI and MAI can be suppressed by the carrier and timing recovery loops in order to guarantee acceptable performance. A joint weighted least squares (WLS) algorithm is adopted for this purpose, which has been shown to estimate $\mathrm{CFO}$ and SCO more accurately than the LS synchronization error estimation algorithm [17] in both stationary and non-stationary time-varying channels [18]. In practice, the carrier frequency and the sampling clock frequency are often generated from the same oscillation source. Thus, CFO and SCO is related by a constant, making the estimation simpler.

If the oscillator deviation is denoted by $\delta$, then the CFO can be expressed as $\epsilon=\delta \times f_{c} T$, where $f_{c}$ is the carrier frequency and $T$ is the symbol time. Assuming that $M+1$ is the number of pilot subcarriers and $-M / 2 \leq m \leq M / 2$, the WLS estimate for $\delta$ is then given by

$$
\hat{\delta}=\frac{\sum_{m} w_{m} \theta_{m}\left(m D+f_{c} T\right)}{2 \pi \frac{N+N_{g}}{N} \sum_{m} w_{m}\left(m D+f_{c} T\right)^{2}}
$$

where $D$ is the spacing of pilot subcarriers, and $\theta_{m}$ and $w_{m}$ are the phase difference and channel power gain at the $m$ th pilot subcarrier, respectively. When the carrier frequency is much higher than the subcarrier spacing, the above estimation formula can be further simplified to

$$
\hat{\delta} \approx \frac{\sum_{m} w_{m} \theta_{m}}{2 \pi \frac{N+N_{g}}{N} f_{c} T \sum_{m} w_{m}} .
$$

With the synchronization error estimation, a loop filter, a numerical-controlled oscillator, and a phase derotator compensate the CFO in the time domain. Likewise, adjustments in FFT window and channel estimates are made according to the derived SCO estimate.

Final detection includes channel estimation, equalization, and despreading of the equalized subcarrier data. The receiver provides two modes of channel estimation and switches between them according to channel variation. When the channel is stationary, LS channel estimation [19] from the training symbol is adopted. In fast-fading channels, a channel estimator which can track time-varying channel frequency response based on comb-type pilot subcarriers is required. Frequency-domain channel interpolators are widely adopted due to hardware simplicity [20], [21]. Time-domain discrete Fourier transform (DFT)-based windowing algorithms have also been proposed to provide better estimation results than conventional interpolation with higher complexity of the DFT and inverse DFT (IDFT) operations [6], [22], [23]. A frequency-domain channel interpolator with a time-domain raised-cosine window and a phase rotation term is proposed to accommodate channel impulse response, noise and aliasing effects. Not only more accurate estimates can be derived than most of the time-domain DFT-based windowing algorithms, but also less hardware resources are utilized without the DFT and IDFT operations by the proposed interpolator [24].

The raised-cosine coefficient of the proposed frequency-domain interpolation takes the form of

$$
W_{l}=\frac{\sin (\pi l / D)}{\pi l / D} \cdot \frac{\cos (\pi \beta l / D)}{1-4 \beta^{2}(l / D)^{2}} \cdot e^{-j 2 \pi d l / N}
$$

where $W_{l}$ is the $l$ th interpolation coefficient; $\beta$ is the roll-off factor; and $d$ is the time shift, which is decided by the worst-case channel delay spread. The channel gain estimates in the data subcarriers are then given by

$$
\begin{aligned}
\hat{H}_{k}= & \sum_{m=\lfloor k / D\rfloor-2}^{\lfloor k / D\rfloor+3} \tilde{H}_{m D} W_{k-m D} \\
= & e^{-j 2 \pi d k / N} \sum_{m=\lfloor k / D\rfloor-2}^{\lfloor k / D\rfloor+3}\left(\tilde{H}_{m D} e^{j 2 \pi d m D / N}\right) \\
& \cdot \frac{\sin (\pi(k-m D) / D)}{\pi(k-m D) / D} \\
& \cdot \frac{\cos (\pi \beta(k-m D) / D)}{1-4 \beta^{2}((k-m D) / N)^{2}}
\end{aligned}
$$

where $\tilde{H}_{m D}$ is the channel response of the $m$ th pilot subcarrier.

To gather most of the signal energy spread across the subcarriers, the receiver first equalizes the subcarrier data and then despreads them to obtain the final decision. As in the traditional multi-carrier systems, the data on each subcarrier of the MC-CDMA signal is assumed to suffer only flat fading and thus can be compensated by a simple one-tap FEQ. For this FEQ, we use the threshold orthogonality restoring combining (TORC) method [25], which strikes a balance between complexity and performance. In this method, noise enhancement in deeply fading subcarriers is avoided while the orthogonality among different users is restored. 
TABLE II

SIGNAL WORD LENGTH OF MAJOR FUNCTIONAL BLOCKS

\begin{tabular}{|l|c|l|c|}
\hline Signal & Word-Length & Signal & Word-Length \\
\hline ADC Output & 12 & FFT Twiddle Factor & 12 \\
\hline Derotator Output & 14 & CORDIC ATAN Input & 12 \\
\hline FFT Output & 16 & CORDIC ATAN Output & 9 \\
\hline Channel Estimator Output & 14 & NCO Phase & 10 \\
\hline TORC Output & 12 & NCO Output & 10 \\
\hline Despreading Output & 14 & WLS Estimation Output & 21 \\
\hline
\end{tabular}

In TORC, the equalizer coefficient $\left(G_{k}\right)$ for subcarrier $k$ can be expressed as

$$
G_{k}= \begin{cases}1 / \hat{H}_{k}, & \text { if }\left|\hat{H}_{k}\right| \geq h_{\mathrm{THR}} \\ \hat{H}_{k}^{*} /\left|\hat{H}_{k}\right|, & \text { if }\left|\hat{H}_{k}\right|<h_{\mathrm{THR}}\end{cases}
$$

where $h_{\mathrm{THR}}$ is a threshold, below which a subcarrier is deemed in deep fade. Finally, the equalized signals are despread and the desired user's signal is obtained.

\section{ARChiteCture AND CirCuit Design}

In the following, the architecture and circuit design of a lowpower downlink baseband receiver will be described. Then, the effectiveness of this design in hardware saving and power saving will be presented.

\section{A. Word Length Optimization}

The downlink cellular communication baseband receiver integrates synchronization and detection modules that support multiple users as well as constellations up to 64-QAM. As such, its complexity can be quite high and so signal word lengths in this receiver must be optimized. Such design optimization not only reduces hardware complexity, but also guarantees acceptable system performance. Toward this end, the signal-to-noise ratio (SNR) at the despreader output (before slicer or soft-input error correcting decorder) is adopted as the performance metric and it is defined as

$$
\begin{aligned}
\mu_{p} & =\frac{1}{N_{p}} \sum_{i=0}^{N_{p}-1} \text { complex }\left(\left|\operatorname{Re}\left\{S_{i}\right\}\right|,\left|\operatorname{Im}\left\{S_{i}\right\}\right|\right) \\
\sigma_{p}^{2} & =\frac{1}{N_{p}-1} \sum_{i=0}^{N_{p}-1}\left(\operatorname{Re}\left\{S_{i}\right\}^{2}+\operatorname{Im}\left\{S_{i}\right\}^{2}-\left|\mu_{p}\right|^{2}\right) \\
\mathrm{SNR} & =10 \log _{10}\left(\frac{\sum_{p=0}^{P-1}\left|\mu_{p}\right|^{2} N_{p}}{\sum_{p=0}^{P-1} \sigma_{p}^{2} N_{p}}\right)
\end{aligned}
$$

where $P$ is the number of symbols in the first quadrant of the transmitted constellation, e.g., in the 16-QAM, $P=4$, and $S_{i}$ is the desired user data after despreading. $N_{p}$ is the number of data belonging to the $p$-th symbol after being sliced. $\operatorname{Re}\{\cdot\}$ and $\operatorname{Im}\{\cdot\}$ is the real part and the imaginary part of its argument respectively. The specified implementation loss due to quantization error is set to be smaller than $0.5 \mathrm{~dB}$ when the symbol error rate is at $10^{-5}$. Signal word lengths of several key signals in the receiver are listed in Table II.

\section{B. FFT Module}

FFT is a key component in multi-carrier transceivers. It is both computation intensive and communication intensive. The FFT size in our application is quite large, therefore we use the radix-2/4/8 algorithm [26] to accomplish the 1024-point FFT (see Fig. 3). With the radix-2/4/8 algorithm, the FFT module requires only three complex multipliers and associated twiddle factor tables. Complex multipliers are realized in three real multiplications and five real additions/subtractions [27]

$$
\begin{array}{r}
(A+j B)(C+j D)=[C \times(A-B)+B \times(C-D)] \\
+j[D \times(A+B)+B \times(C-D)] .
\end{array}
$$

Furthermore, symmetry in the sine/cosine functions is exploited and the look-up table stores only the sine and cosine values from 0 to $\pi / 4$.

For data scheduling in the FFT architecture, several delay buffers of various lengths are required. Longer delay buffers are implemented with two single-port SRAM modules, which consume less area and power than one two-port SRAM module. Nonetheless, delay buffers shorter than 64 are implemented by D flip-flops that are configured in a pointer first-in-first-out (FIFO) configuration [28]. A ring counter with only one active cell is used to activate one word for read-out and one word for write-in. This arrangement greatly reduces data transitions and thus power consumption. More power can be saved by using one ring counter for several delay buffers, as depicted in Fig. 4.

The decimation-in-frequency FFT outputs are in the bit-reverse address order. They need to be rearranged into the normal order for further frequency-domain signal processing such as channel interpolation and despreading. Conventionally, two $\mathrm{N}$-word SRAM modules working in the ping-pong fashion are used [6]. One is being written in the bit-reverse order, while data in the other is read out in the normal order. After one cycle of $N$ words, the two SRAM modules exchange roles. We propose a more complex, yet area-efficient implementation using two SRAM modules each with $N / 2$ words, saving $50 \%$ of the memory.

We will use an 8-point FFT and two 4-word SRAM modules as an example. Refer to Fig. 5(a) and note that gray pockets with numbers denote FFT outputs with the number indicating the frequency-domain indices.

- FFT output data of the same subcarrier index are not always stored at the same position. Instead, they are stored in the memory word whose data have just been read out. Let each FFT output data of the first symbol be stored using the address (iBRAdd) that happens to be the same as its subcarrier index. On the other hand, when the second symbol arrives, in order to read the first symbol in normal order, the address (iBRAdd) must be given in the normal order. Similarly, when the third symbol arrives, the address must be in the bit-reverse order again, and so on.

- The two SRAM modules (Bank 0 and Bank 1) are both single-port memory, leaving each of them only capable of either one read or one write operation in one clock cycle. In order to avoid data loss, we have to perform the read operation before the write operation. So two FFT outputs are written to the two modules in each odd clock cycle and the 

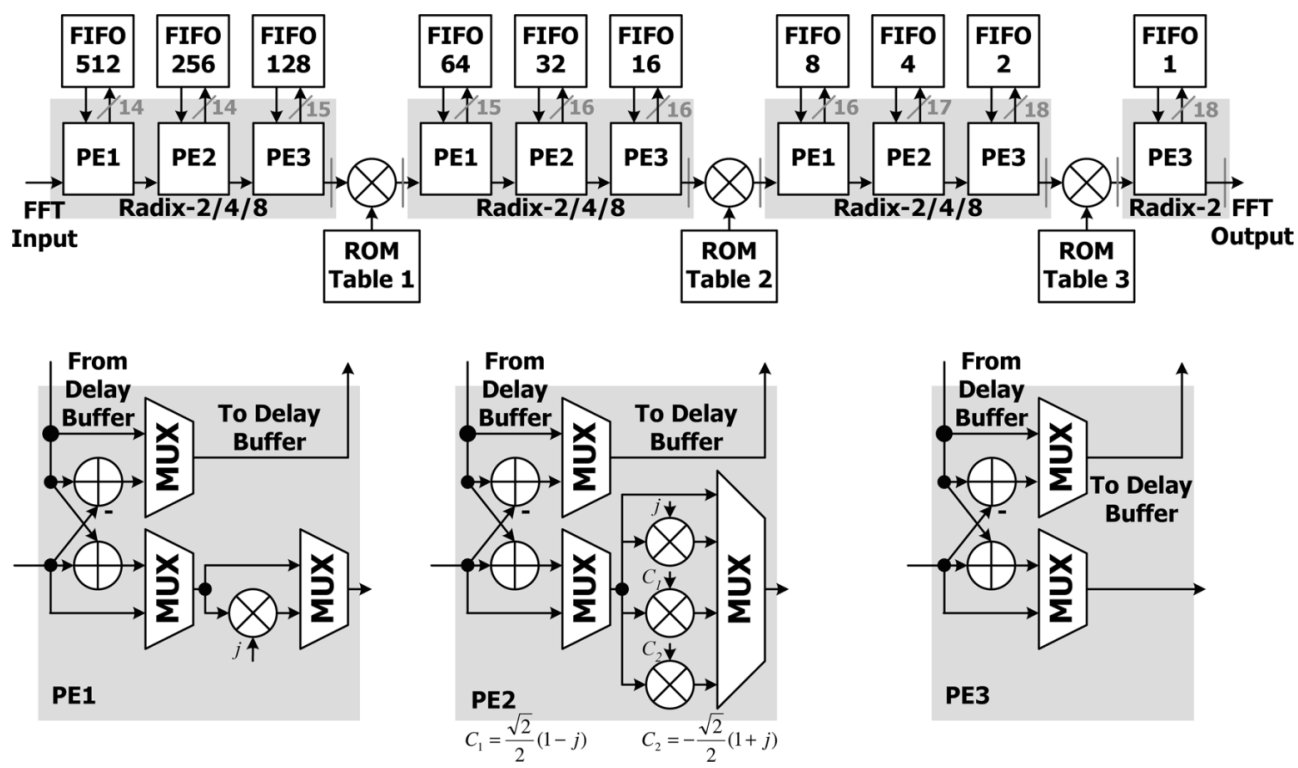

Fig. 3. Architecture of the radix 2/4/8 FFT module.

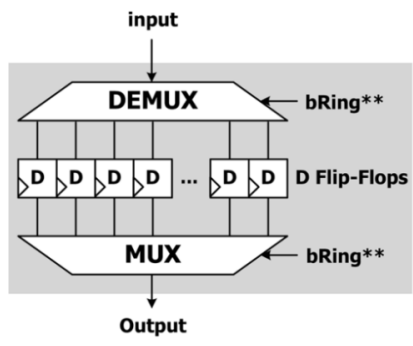

(a)

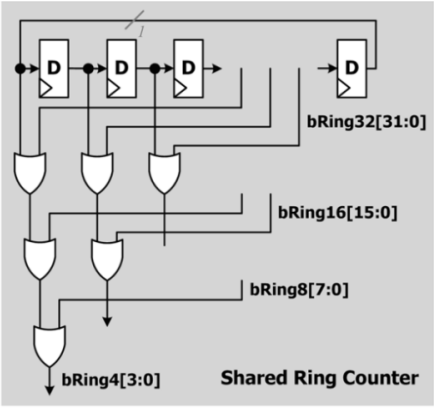

(b)
Fig. 4. (a) Pointer-based delay buffer. (b) Sharing one ring counter among several delay buffers.

even clock cycles are reserved for read-out. Consequently, the write enable signals $\overline{\mathrm{WEN}}$ for the two banks are toggled in every clock cycle.

- In order to avoid retrieving two data from the same bank, Bank 0 stores those memory words whose addresses (iBRAdd) have even sum of the most significant bit (MSB) and the least significant bit (LSB), while Bank 1 stores the other half of the memory words. Since two FFT outputs are written at once, one of them must be delayed for one clock cycle. When the address (iBRAdd) is issued in the normal order, the MSB is used to direct the two FFT outputs to the corresponding memory bank. On the contrary, the LSB of the address is used (CtrIn) when the address is in the bit-reversed order. Similar control scheme for output redirection is adopted (CtrOut).

- Note that if the frequency-domain data are intended to be output in the normal order but the negative-band data must be processed prior to the positive-band data $(4,5,6,7,0,1,2,3$ in this example), the same idea can still be applied. However, instead of two alternative address sequences (iBRAdd), four sequences will be generated.

The block diagrams of the control logic and the whole FFT output reordering module are illustrated in Fig. 5(b).

\section{Arctangent Module}

In multi-carrier communication systems, the arctangent function is commonly used for signal processing in the receiver because the phase of both the time-domain and frequency-domain complex signals provides important information about the synchronization errors. One arctangent module is shared among initial fractional CFO acquisition, fine symbol boundary detection, and WLS CFO/SCO estimation. In these three blocks, it is not necessary to compute the arctangent function in one clock. In light of the above, the COordinate Rotation DIgital Computer (CORDIC) hardware that computes the arctangent function in several clock cycles is adopted [29]. In this design, only a small $8 \times 10 \mathrm{ROM}$ is needed. The block diagrams of the CORDIC-based arctangent module are illustrated in Fig. 6(a). However, due to different dynamic ranges of input complex signals, the essential 12 MSBs of the 16-bit FFT outputs are selected to be fed into the shared CORDIC module so as to guarantee the resolution of the arctangent function. The bit transition from " 0 " to " 1 " in positive numbers and " 1 " to " 0 " in negative numbers of five MSBs are checked. The block diagram is shown in Fig. 6(b).

\section{Channel Estimation}

As mentioned earlier, we provide two modes of channel estimation. One is the LS channel estimator for the slow-fading channels, which simply examines the complex gain between the received signals and the transmitted signals. Then, phase rotation caused by the SCO effect is also corrected. The other is the frequency-domain channel interpolator. In the mobile cellular environments, the received signals may suffer fast fading and it is very important to attain accurate channel estimation in fastfading channels. Normally, the receiver uses LS channel estimation. If the LS channel estimation can not track channel variation, frequency-domain channel interpolation is then activated.

The frequency-domain interpolation formula is given in (4). The interpolator circuit (see Fig. 7) takes in the channel responses of pilot subcarrier, $\tilde{H}_{(m+3) D},\lfloor k / D\rfloor=m$ and apply 


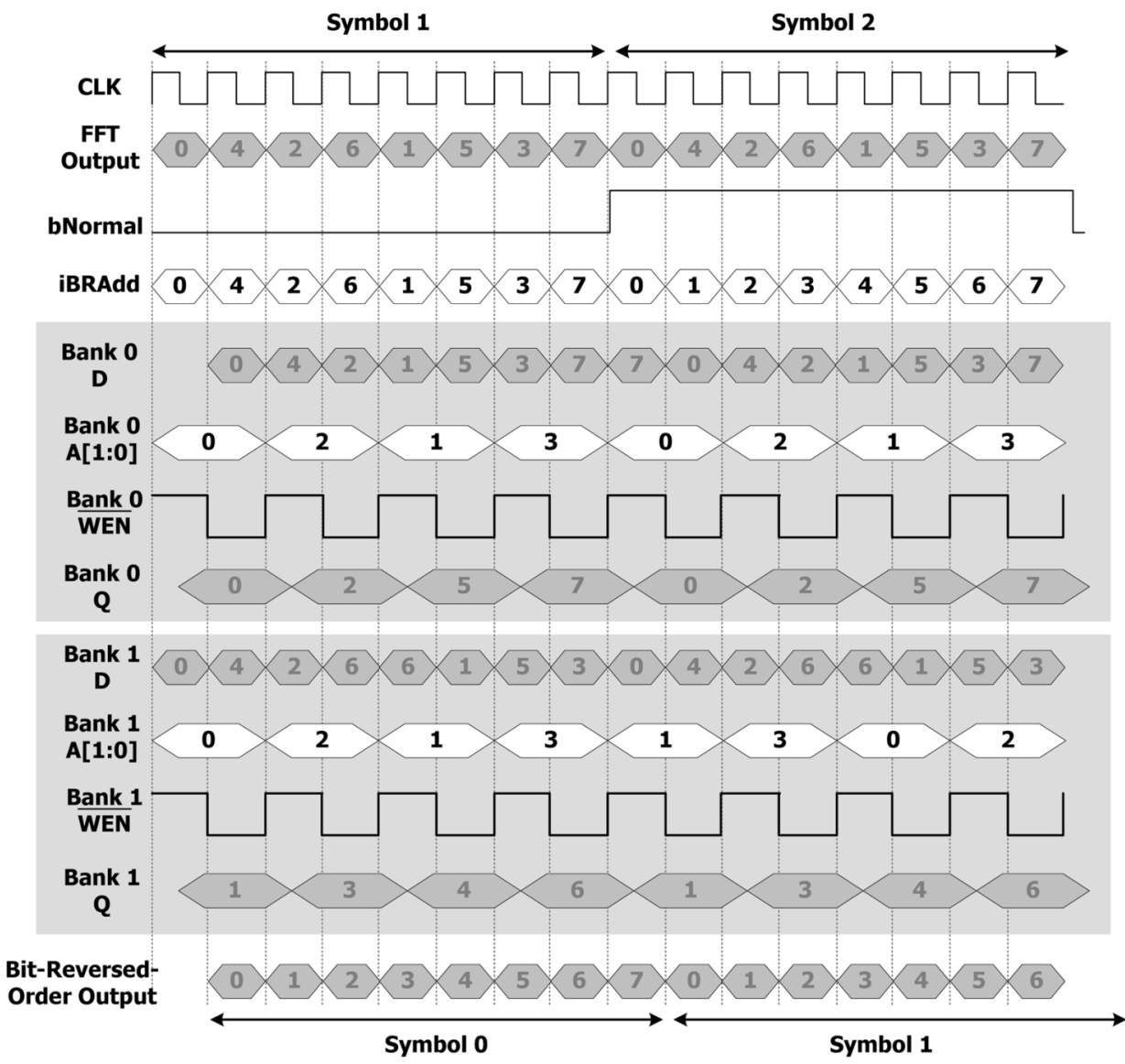

(a)
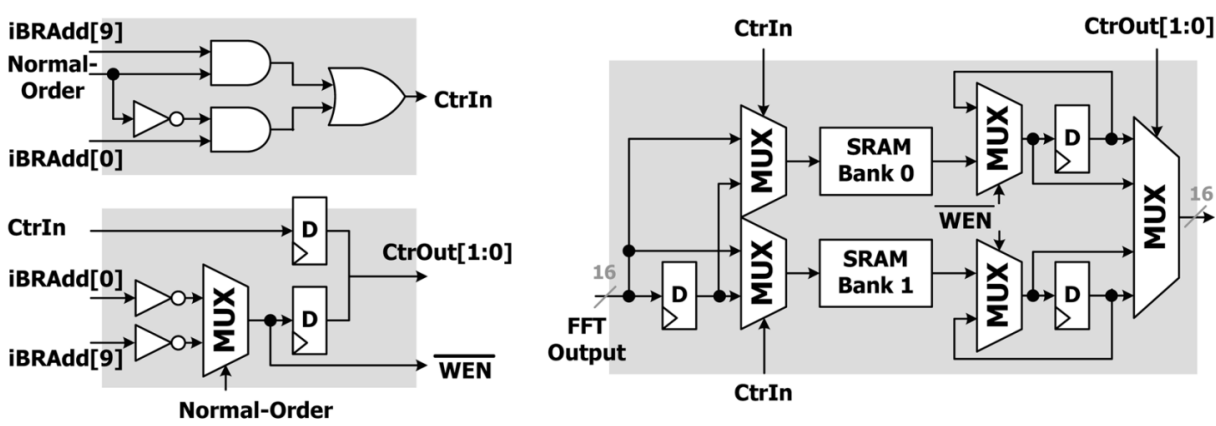

(b)

Fig. 5. (a) Timing diagram of the reordering block. (b) Block diagram of the control logic for the reordering SRAM.

phase rotation. This is followed by a six-tap real-coefficient interpolator. Then, the channel estimate of $k$ th data subcarrier is computed by multiplying another phase rotation term.

\section{E. Equalizer}

The TORC equalizer coefficients in (5) can be reformulated as

$$
G_{k}= \begin{cases}\frac{1}{\left|\hat{H}_{k}\right|} \angle\left(\hat{H}_{k}^{*}\right), & \text { if }\left|\hat{H}_{k}\right| \geq h_{\mathrm{THR}} \\ \angle\left(\hat{H}_{k}^{*}\right), & \text { if }\left|\hat{H}_{k}\right|<h_{\mathrm{THR}}\end{cases}
$$

For better hardware reuse, we use the CORDIC algorithm to implement the TORC equalizer and its architecture is depicted in Fig. 8. In this scheme, the estimated complex channel response of the $k$ th subcarrier is rotated back to the $\mathrm{x}$-axis in the complex plane to obtain the phase of the channel response. Meanwhile, the received frequency-domain data of that subcarrier is also rotated by the same amount. Without explicitly deriving the channel phase and with only eight steps of micro-rotation in the CORDIC algorithm, the frequency-domain data are equalized in phase. These eight micro-rotation steps are implemented in eight stages of the CORDIC hardware that consists of two dedicated shifters and two adders/subtractors. In addition, one more initial stage is needed to rotate the input by multiples of $\pi / 2$ so that the resultant output will have a phase between 0 and $\pi / 2$, which is necessary for the CORDIC algorithm. Two stages of pipeline registers are inserted between the above nine stages. The circuits of each micro-rotation stage are also depicted. In each such stage, four adders/subtractors are required and the 


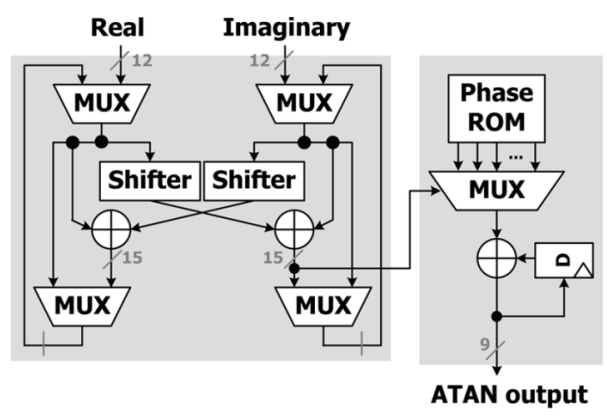

(a)

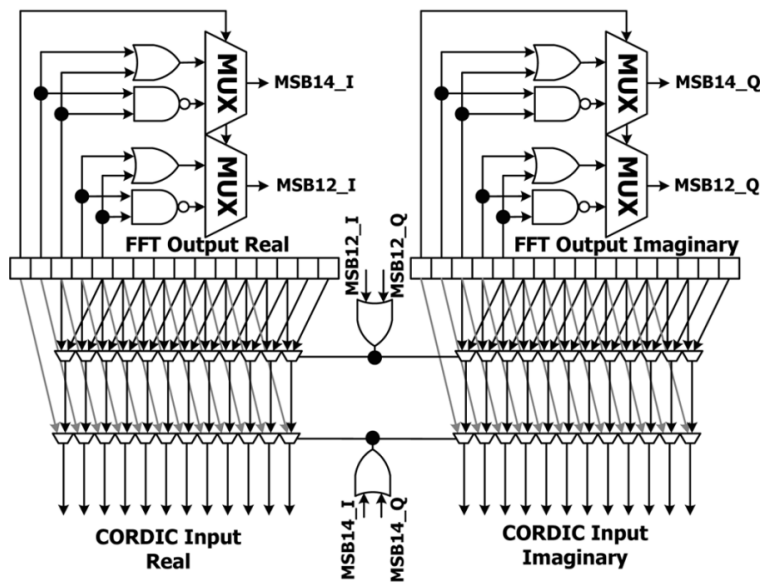

(b)

Fig. 6. Block diagram of (a) the CORDIC-based arctangent function and (b) the real 12-MSB selection.

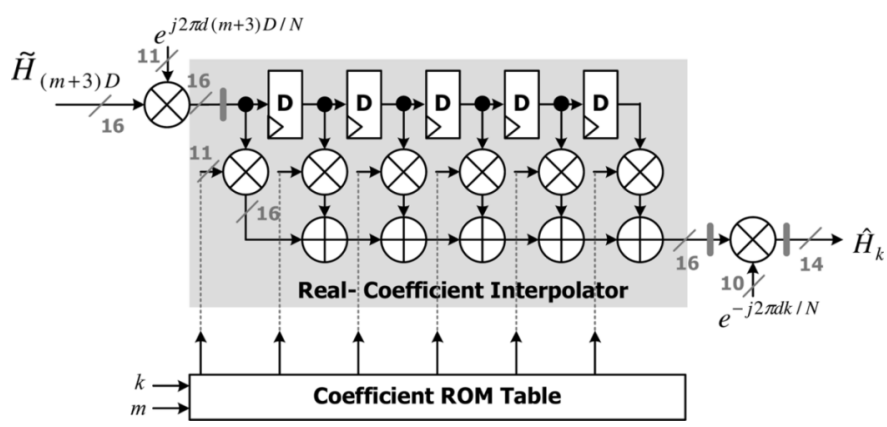

Fig. 7. Hardware implementation of the frequency-domain channel interpolation.

shifters involve only sign extension since the number of bit-shift is fixed in each stage.

After phase compensation, the magnitude of the signal needs also to be properly scaled. No additional hardware is required for the calculation of the channel magnitude, $\left|\hat{H}_{k}\right|$, since the CORDIC hardware computes this magnitude as the complex channel gain is rotated back to the $\mathrm{x}$-axis. A multiplexer is then used to decide the divisor based on the value of $\left|\hat{H}_{k}\right|$ (ORC or EGC). This CORDIC-based TORC equalizer architecture requires only eight sets of four adders/subtractors. On the other hand, direct implementation of (5) requires one complex multiplier, two square functions, one adder, plus one square root function.

\section{F. Saving in Complexity and Power}

In Table III, we summarize the hardware saving resulting from the techniques mentioned above. Design techniques which have been used in other OFDM receivers for the same function are compared. According to the SRAM compiler report, the single-port SRAM solution to the delay buffers saves about $50 \%$ to $70 \%$ of the die area than the dual-port SRAM solution. The special bit-reverse reordering block that we proposed only uses half SRAM size than that needed in the traditional ping-pong style reordering. The CORDIC-based TORC equalizer architecture also saves about $40 \%$ of the area because it uses only adders instead of complex multipliers.

The CORDIC ATAN block requires two adders for coordinate rotation and one adder for phase accumulation. In contrast, conventional ATAN structure involves one divider and a look-up table. The proposed frequency-domain channel interpolator also contributes big saving in hardware complexity. This scheme can do without the FFT/IFFT operations that transform pilot information from frequency domain to the time domain and then back to the frequency domain again, which are needed in the time-domain channel estimation algorithms [6], [23]. In addition, due to shorter latency in the frequency-domain interpolator, the proposed scheme can further save about $95 \%$ of the buffers that store data subcarrier signals for equalization.

During the architecture design, we have re-used as many modules in the receiver as possible. For instance, SRAM modules used for delay buffers in the symbol boundary detection block and in the FFT block are time-shared. Also, only one CORDIC ATAN module is shared among fractional CFO acquisition, fine symbol boundary detection, and joint estimation of residual synchronization errors.

Fig. 9 illustrates the step-by-step power reduction before and after each power-saving technique. The power figures are estimated by either a SRAM compiler or a power compiler. As can be seen from the figure, special memory block design of singleport SRAMs and pointer-based delay buffers reduces about $13 \%$ of the power compared to two-port SRAMs and shift registers. The new channel estimator saves even more power due to elimination of the extra FFT and IFFT operations in the time-domain DFT-based algorithms [6], [23]. The TORC equalizer can also save some power consumption than direct implementation of (5). The proposed chip consumes only about $18 \%$ of the power dissipated by the receiver without all the design techniques.

\section{EXPERIMENTAL RESULTS}

The entire MC-CDMA baseband receiver is integrated and implemented in $0.18-\mu \mathrm{m}$ one-poly and six-metal CMOS technology. The chip is designed using a cell-based flow. The gate count is about $336 \mathrm{~K}$ and the transistor count is around 2.1 millions. With various efforts to share the SRAM modules among functional blocks, only 12 SRAM modules ( $81 \mathrm{~K}$ bits) are used and they are placed near the core boundary to prevent malfunction caused by IR drops. To facilitate testing, scan chains, test buses, and test pins are inserted and memory build-in-self-test (BIST) circuits are incorporated in all SRAM modules. The core size of this chip is $2.6 \mathrm{~mm} \times 2.6 \mathrm{~mm}$ and the chip photograph is shown in Fig. 10. 


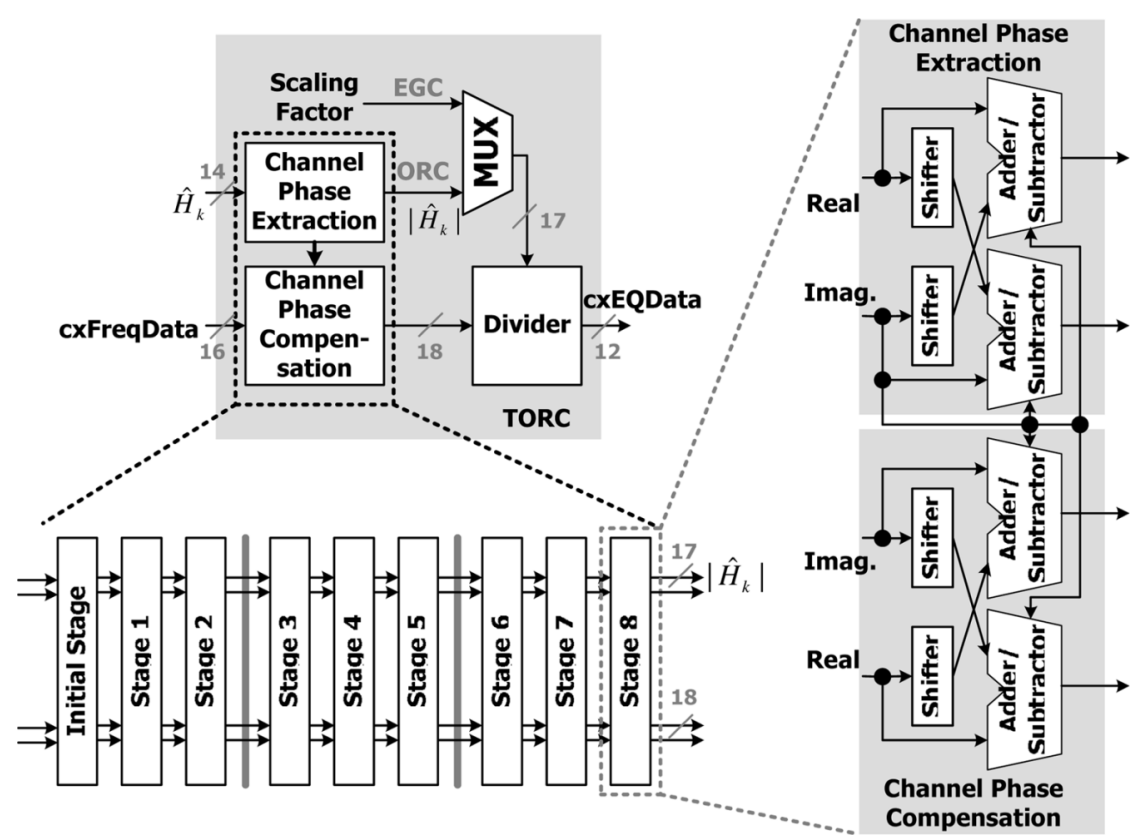

Fig. 8. Hardware design of the TORC equalizer.

TABLE III

HARDWARE SAVING

\begin{tabular}{|c|c|c|}
\hline \multicolumn{3}{|c|}{ Hardware Saving } \\
\hline & This Work & Other Designs \\
\hline \multirow{2}{*}{$\begin{array}{c}\text { Delay Buffer } \\
512 \times 28\end{array}$} & Single-Port SRAM & Dual-Port SRAM [6][30] \\
\hline & $194928\left(\mu \mathrm{m}^{2}\right)$ & $332010\left(\mu \mathrm{m}^{2}\right)$ \\
\hline \multirow{2}{*}{$\begin{array}{c}\text { Delay Buffer } \\
256 \times 28\end{array}$} & Single-Port SRAM & Dual-Port SRAM [6][30] \\
\hline & $131768\left(\mu \mathrm{m}^{2}\right)$ & $209202\left(\mu \mathrm{m}^{2}\right)$ \\
\hline \multirow{2}{*}{$\begin{array}{l}\text { Bit-Reverse } \\
\text { Reordering }\end{array}$} & Proposed & Ping-Pong style [6][23] \\
\hline & $346904\left(\mu \mathrm{m}^{2}\right)$ & $618411\left(\mu \mathrm{m}^{2}\right)$ \\
\hline \multirow{2}{*}{ TORC } & Proposed & Direct Implementation \\
\hline & $157102\left(\mu \mathrm{m}^{2}\right)$ & $212580\left(\mu \mathrm{m}^{2}\right)$ \\
\hline \multirow{2}{*}{ ATAN } & CORDIC & Look-up Table \\
\hline & 3 adders, 8-word ROM. & 1 divider, 128 -word ROM. \\
\hline \multirow{2}{*}{$\begin{array}{l}\text { Channel } \\
\text { Estimator }\end{array}$} & Proposed & Time-Domain [23] \\
\hline & $\begin{array}{l}12 \text { multipliers, } 10 \\
\text { adders, } 150 \text { buffers. }\end{array}$ & $\begin{array}{l}\text { 32-point IFFT, 1024-point } \\
\text { FFT, } 4096 \text { buffers. }\end{array}$ \\
\hline
\end{tabular}

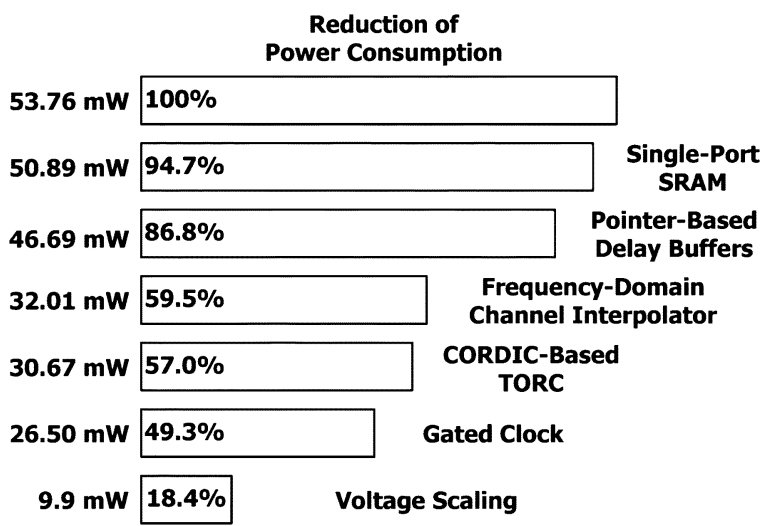

Fig. 9. Power saving.

The fabricated chip was tested using a pattern generator and a logic analyzer. The test signal is made up of 32 users with 16-QAM modulation and a spreading factor of 64 . In addition, some synchronization errors are also included. Fig. 11(a) shows

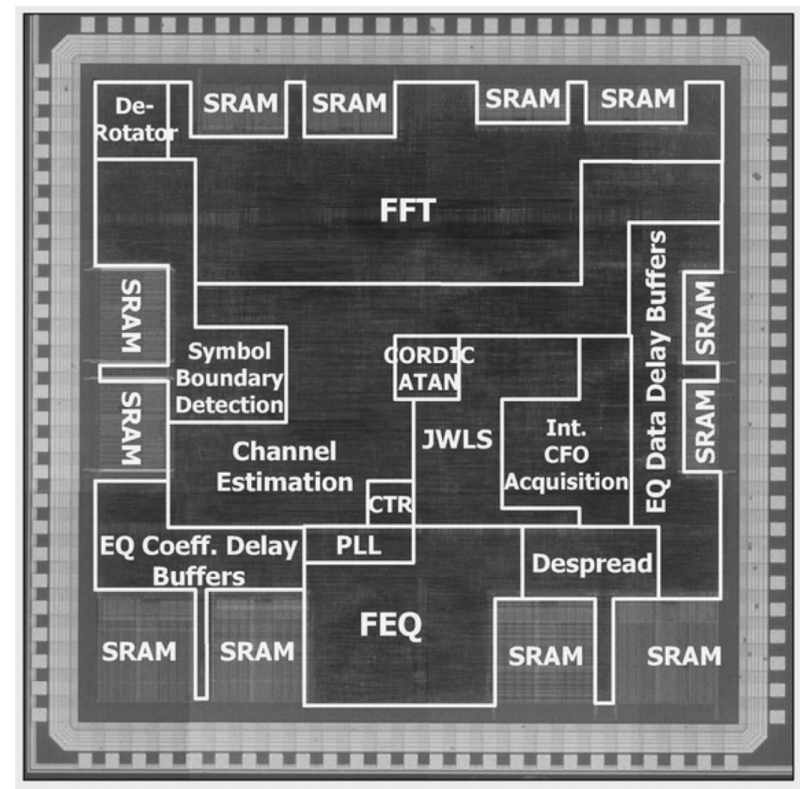

Fig. 10. Die photo of the proposed MC-CDMA downlink baseband receiver IC.

the power dissipation versus different supply voltages at the nominal operating frequency, $5.76 \mathrm{MHz}$ in different channel conditions. When operating in mobile channels, the receiver turns on the channel interpolation function so that a little more power is consumed than in stationary channels. Thanks to power saving techniques and the voltage scaling, this chip consumes only $9.9 \mathrm{~mW}$ at the nominal $5.76 \mathrm{MHz}$ from a supply voltage of $1.1 \mathrm{~V}$. The maximum operating frequency versus voltage is also indicated in the same figure. In the design, we inserted pipeline registers to keep the critical path delay below $20 \mathrm{~ns}$ at $1.8 \mathrm{~V}$. From the figure, it is clear that malfunction of the chip at low voltage is not caused by the critical path but rather by the failure of SRAM modules. We have to emphasize that the low nominal operating frequency, equal to the sample frequency and well 


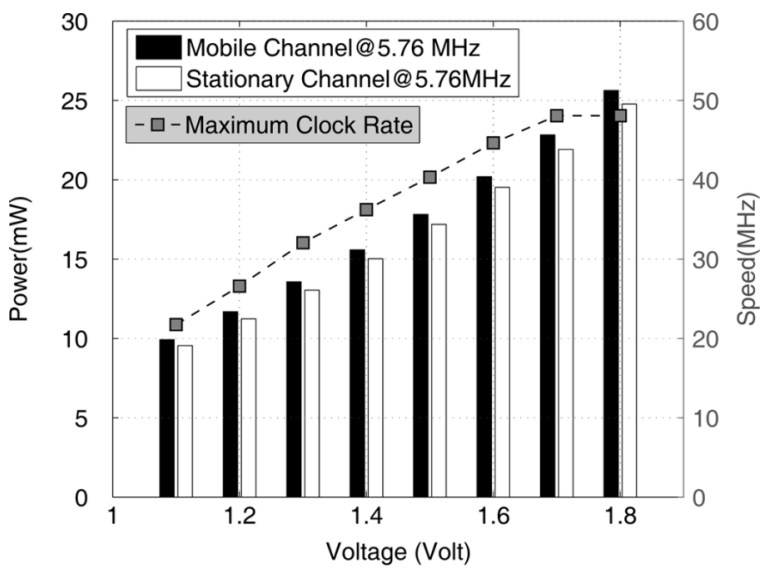

(a)

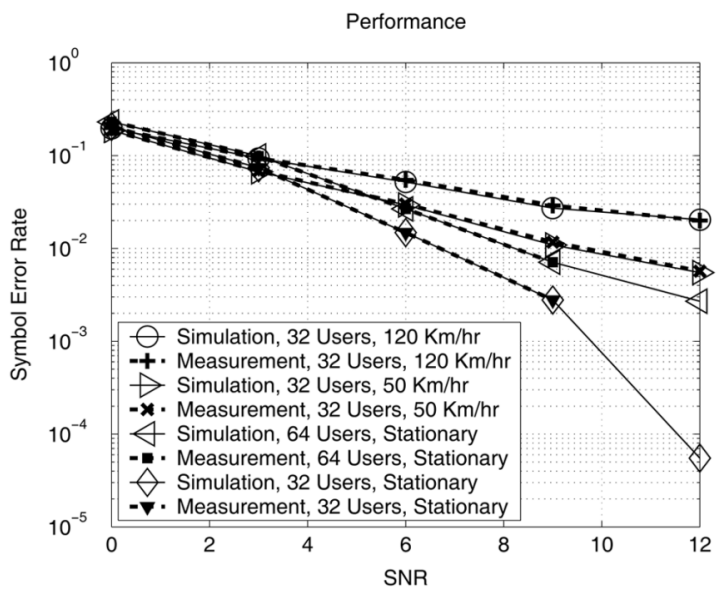

(b)

Fig. 11. (a) Power dissipation versus supply voltage at nominal operating frequency and maximum clock frequency versus supply voltage. (b) Functional simulation and measurement performances of the proposed downlink MC-CDMA receiver.

TABLE IV

SUMMARY OF THE MC-CDMA CHIP

\begin{tabular}{|l|l|c|}
\hline \multicolumn{2}{|l|}{ Technology } & $0.18 \mu \mathrm{m}$ 1P6M CMOS \\
\hline \multicolumn{2}{|l|}{ Package } & CQFP-100 \\
\hline \multirow{2}{*}{ Size $\left(\mathrm{mm}^{2}\right)$} & Core & $2.6 \times 2.6$ \\
\cline { 2 - 3 } & Chip & $3.16 \times 3.16$ \\
\hline Gate Count & 335823 \\
\hline Transistor Count & 2093944 \\
\hline SRAM Size (bits) & $81 \mathrm{~K}$ \\
\hline \multicolumn{2}{|l|}{ Power Consumption } & $9.9 \mathrm{~mW} @ 1.1 \mathrm{~V}, 5.76 \mathrm{MHz}$ \\
\hline Max. Oscillator Deviation (ppm) & 10 \\
\hline
\end{tabular}

afforded by the $0.18-\mu \mathrm{m}$ CMOS technology, is contributed by these low-power design techniques, especially the proposed frequency-domain channel interpolation algorithm. A summary of MC-CDMA baseband receiver IC is given in Table IV.

Its receiving performance is also tested in multi-path fading channels. The channel model used is the typical urban powerdelay profile with maximum excess delay of $2.14 \mu$ s specified in the 3GPP technical document [9]. A total of 20 paths ensure the realistic frequency-domain correlation property. The modified Jake's model is adopted to generate the time-varying channel gain [31]. The CFO and the SCO are also injected and set to 2.2
TABLE V

IC PERFORMANCE COMPARISON

\begin{tabular}{|l|l|l|}
\hline & Zou $[23]$ & This Work \\
\hline Technology & $0.18 \mu \mathrm{m}$ & $0.18 \mu \mathrm{m}$ \\
\hline Power & $32 \mathrm{~mW}$ & $9.9 \mathrm{~mW}$ \\
\hline Supply Voltage & $1.8 \mathrm{~V}$ & $1.1 \mathrm{~V}$ \\
\hline Sample Frequency & $6.5 \mathrm{MHz}$ & $5.76 \mathrm{MHz}$ \\
\hline Clock Frequency & $52 \mathrm{MHz}$ & $5.76 \mathrm{MHz}$ \\
\hline Throughput & $8.192 \mathrm{Mbps}$ & $21.7 \mathrm{Mbps}$ \\
\hline Normalized Energy per Mega Bits & $1.29 \mathrm{~mJ}$ & $0.46 \mathrm{~mJ}$ \\
\hline
\end{tabular}

subcarrier spacing and $-6.2 \mathrm{ppm}$, respectively. In Fig. 11(b), the effect of MAI is depicted when the system is either fully loaded or half-loaded. A spreading factor of 64 is used and 16-QAM is adopted. Due to the memory depth limitation of the pattern generator, the measurement results can only be depicted above $10^{-3}$. It is clear that the chip's symbol error rate (SER) performance coincides with the results obtained in functional simulation. Note that with 32 active users and $226 \mathrm{kbps}$ per user, the receiver can reach an uncoded SER on the order of $10^{-5}$ given enough SNR. If the system is fully loaded, then severe MAI causes the uncoded SER around $10^{-3}$. Also the receiver's performances are compared under different mobility. With only 32 active users, this receiver chip attains satisfactory uncoded SER around $10^{-2}$ even at the highest speed, $120 \mathrm{~km} / \mathrm{h}$.

Table V lists the comparisons between our MC-CDMA chip and a similar work [23], implementing an OFDM receiver in a 6.5-MHz channel with 1024 subcarriers. Note that the two receivers have distinct functions and a fair comparison between them is hard to come by. Nevertheless, they both have a 1024point FFT module and a channel estimation block, which represent a significant portion of their respective power consumption. From the table, we can see the proposed channel estimator avoids overdriving clock to a higher rate as in [23] and thus the proposed chip can operate at a lower voltage and at a clock rate equivalent to sample frequency. The highest throughput is achieved for 64-QAM constellation and a single user taking the training symbol overhead into account. The normalized energy per mega bits is obtained by

$$
\begin{array}{r}
\frac{\text { Energy }}{10^{6} \text { Bits }}=\text { Power } \times \frac{1.1^{2}}{\text { Voltage }^{2}} \times \frac{5.76 \times 10^{6}}{\text { Sample Frequency }} \\
\times \frac{10^{6}}{\text { Throughput }} .
\end{array}
$$

We can see the proposed chip has only $36 \%$ power consumption compared to the previous work.

In conclusion, the proposed MC-CDMA downlink baseband receiver chip not only provides a low-power solution but also is capable of broadband wireless communication in fast-fading mobile channels.

\section{CONCLUSION}

A downlink baseband receiver suitable for MC-CDMA systems is presented in this paper. A symbol boundary detector, a carrier frequency recovery loop, a timing recovery loop, a channel estimator, and a combining and despreading module are all integrated in this single chip. With the WLS estimation 
algorithm for the $\mathrm{CFO}$ and $\mathrm{SCO}$, the synchronization errors are tracked and the ICI is suppressed. The proposed FFT output reordering module and resource sharing save much memory. In addition, the proposed frequency-domain channel interpolator is shown to combine both advantages of the time-domain DFT-based algorithm and the frequency-domain interpolator and does not have their drawbacks. Moreover, the CORDIC-based TORC equalizer architecture reduces hardware complexity without any performance degradation. This receiver was fabricated in a $0.18-\mu \mathrm{m} \mathrm{CMOS} \mathrm{technology} \mathrm{and} \mathrm{can} \mathrm{operate}$ at $5.76 \mathrm{MHz}$ from $1.1-\mathrm{V}$ supply voltage while drawing $9.9 \mathrm{~mW}$. With all these features, this MC-CDMA baseband receiver can play an important role in supporting future high-rate mobile multiple-access communication over multipath fading channel.

\section{ACKNOWLEDGMENT}

The authors greatly appreciate the Chip Implementation Center (CIC) of the National Science Council, Taiwan, R.O.C., for the fabrication of the proposed chip.

\section{REFERENCES}

[1] Technical Specification Group Radio Access Network, Feasibility study for orthogonal frequency division multiplexing (OFDM) for UTRAN Enhancement, 3GPP, TR 25.892, V6.0.0, Jun. 2004.

[2] S. Hara and R. Prasad, "Overview of multicarrier CDMA," IEEE Commun. Mag., vol. 35, no. 12, pp. 126-133, Dec. 1997.

[3] 4MORE modem test and demonstration specification IST-4 More, Grenoble, France, 2004 [Online]. Available: http://www.ist-4more.org

[4] Physical layer simulation chain description IST-Matrice, , , 2002 [Online]. Available: http://www.ist-matrice.org

[5] J. Thomson et al., "An Integrated 802.11a baseband and MAC processor," in Dig. Tech. Papers ISSCC, Feb. 2002, pp. 92-93.

[6] W. Eberle et al., " $80-\mathrm{Mb} / \mathrm{s}$ QPSK and 72-Mb/s 64-QAM flexible and scalable digital OFDM tranceiver ASICs for wireless local area networks in the 5-GHz band," IEEE J. Solid-State Circuits, vol. 36, no. 11, pp. 1829-1838, Nov. 2001.

[7] P. Y. Tsai, H. Y. Kang, and T. D. Chiueh, "Design of a baseband transceiver for multi-carrier CDMA communication," EURASIP J. Appl. Signal Process., no. 11, pp. 1645-1655, Jul. 2005.

[8] P. Y. Tsai and T. D. Chiueh, "A 1.1-V 9.9-mW MC-CDMA downlink baseband receiver IC for next-generation cellular communication systems," in Proc. IEEE 2005 Asian Solid-State Circuits Conf., Nov. 2005, pp. 489-492.

[9] Technical Specification Group (TSG) RAN WG4, Deployment Aspects, 3GPP, TR 25.943 V1.2.0.

[10] Digital Audio Broadcasting (DAB); Guidelines and rules for implementation and operation; Part 3: Broadcast network, ETSI TR 101 496-3 V1.1.1, Nov. 2000.

[11] M. Russel and G. L. Stuber, "Interchannel interference analysis of OFDM in a mobile environment," in Proc. IEEE VTC'95, Jul. 1995, vol. 2, pp. 820-824.

[12] Spreading and Modulation (FDD), 3G TR 25.213 v3.3.0, Oct. 1999, 3GPP TSG RAN WG1.

[13] K. Fazel and L. Papke, "On the performance of convolutionally coded CDMA/OFDM for mobile communication system," Proc. IEEE PIMRC'93, pp. 468-472, Sep. 1993, Yokohama, Japan.

[14] T. M. Schmidl and D. C. Cox, "Robust frequency and timing synchronization for OFDM," IEEE Trans. Commun., vol. 45, pp. 1613-1621, Dec. 1997

[15] Y. H. Kim, I. Song, S. Yoon, and S. R. Park, "An efficient frequency offset estimator for OFDM systems and its performance characteristics," IEEE Trans. Vehic. Technol., vol. 50, no. 9, pp. 1307-1312, Sep. 2001.

[16] Y. Kim, S. Choi, C. You, and D. Hong, "BER Computation of an MC-CDMA System with carrier frequency offset," in Proc. 1999 IEEE Int. Conf. Acoust., Speech, Signal Process., Phoenix, AZ, Mar. 1999, pp. 2555-2558.

[17] T. Fujisawa et al., "A single-chip 802.11a MAC/PHY with a 32-b RISC processor," IEEE J. Solid-State Circuits, vol. 38, no. 11, pp. 2001-2009, Nov. 2003.
[18] P. Y. Tsai, H. Y. Kang, and T. D. Chiueh, "Joint weighted least squares estimation of carrier-frequency offset and timing offset for OFDM systems over multipath fading channels," IEEE Trans. Vehic. Technol., vol. 54, no. 1, pp. 211-224, Jan. 2005.

[19] J. J. van de Beek, O. Edfors, M. Sandell, S. K. Wilson, and P. O. Borjesson, "On channel estimation in OFDM systems," in Proc. IEEE 43rd Vehic. Tech. Conf., Jul. 1995, pp. 815-819.

[20] J. Rinne and M. Renfors, "Pilot spacing in orthogonal frequency division multiplexing systems on practical channels," IEEE Trans. Consum. Electron., vol. 42, no. 4, pp. 959-962, Nov. 1996.

[21] S. Coleri, M. Ergen, A. Puri, and A. Bahai, "Channel estimation techniques based on pilot arrangement in OFDM systems," IEEE Trans. Broadcast., vol. 48, no. 3, pp. 223-229, Sep. 2002.

[22] H. Minn and V. K. Bhargava, "An investigation into time-domain approach for OFDM channel estimation," IEEE Trans. Broadcast., vol. 46, no. 4, pp. 240-248, Dec. 2000.

[23] H. Zou and B. Daneshrad, "A $32 \mathrm{~mW}$ self contained OFDM receiver ASIC for mobile cellular applications," in Dig. Tech. Papers Symp. VLSI Circuits, , Jun. 2004, pp. 148-151.

[24] P. Y. Tsai and T. D. Chiueh, "Frequency-Domain Interpolation-Based Channel Estimation in Pilot-Aided OFDM Systems," in Proc. IEEE 59th VTC, Milan, Italy, May 2004, vol. 1, pp. 420-424.

[25] R. Le Gouable and M. Helard, "Performance of single and multi-user detection techniques for a MC-CDMA system over channel model used for HIPERLAN2," in Proc. IEEE Int. Symp. 2000 IEEE 6th Spread Spectrum Tech. Appl., 2000, pp. 718-722.

[26] L. Jia, Y. Gao, J. Isoaho, and H. Tenhunen, "A New VLSI-oriented FFT algorithm and implementation," in Proc. of IEEE ASIC Conf., 1998, pp. $337-341$

[27] A. Wenzler and E. Luder, "New structures for complex multipliers and their noise analysis," in Proc. IEEE Int. Symp. Circuits Syst., May 1995, vol. 2, pp. 1432-1435.

[28] E. K. Tsern and T. H. Meng, "A low power video-rate pyramid VQ decoder," IEEE J. Solid-State Circuits, vol. 31, no. 11, pp. 1789-1794, Nov. 1996

[29] Y. H. Yu, "CORDIC based VLSI architecture for digital signal processing," IEEE Signal Process. Mag., pp. 16-35, Jul. 1992.

[30] C.-C. Wang, J.-M. Huang, and H.-C. Cheng, "A 2 K/8 K mode smallarea FFT processor for OFDM demodulation of DVB-T receivers," IEEE Trans. Consum. Electron., vol. 51, pp. 28-32, Feb. 2005.

[31] P. Dent, G. E. Bottomley, and T. Croft, "Jakes fading model revisited," Electron. Lett., vol. 29, no. 13, pp. 1162-1163, Jun. 1993.

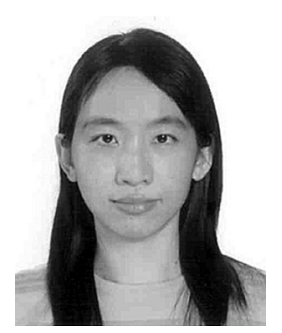

Pei-Yun Tsai (S'02-M'06) received the B.S., M.S., and Ph.D. degrees in electrical engineering from the National Taiwan University, Taipei, Taiwan, R.O.C., in 1994, 1996, and 2005, respectively.

From 1996 to 2000, she worked at ASUStek and participated in the team of optical storage systems. She is currently an Assistant Professor of Electrical Engineering at National Central University, Taoyuan, Taiwan, R.O.C

Prof. Tsai has received the Acer Longtern Award, MXIC Golden Silicon Award, and 1st Asian SolidState Circuit Conference Student Design Contest Outstanding Award in 2005. Her research interests include baseband signal processing algorithms and VLSI design for digital communication systems.

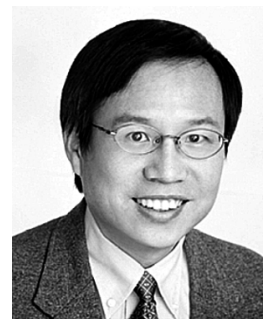

Tzi-Dar Chiueh (S'87-M'90-SM'03) received the B.S. degree in electrical engineering from National Taiwan University, Taipei, Taiwan, R.O.C., and the $\mathrm{Ph} . \mathrm{D}$. degree in electrical engineering from California Institute of Technology, Pasadena, in 1983 and 1989, respectively.

$\mathrm{He}$ is currently a Professor of Electrical Engineering at National Taiwan University. Since August 2004, he has also served as the Director of the Graduate Institute of Electronics Engineering in the same university. His research interests include algorithm, architecture, and integrated circuits for baseband communication systems.

Prof. Chiueh has received the Acer Longtern Award 11 times and the Golden Silicon Award in 2002 and 2005. His teaching efforts were recognized five times by the Teaching Excellence Award from NTU. Prof. Chiueh was the recipient of the Outstanding Research Award from National Science Council, Taiwan in 2004-2006, and was awarded the Himax Chair Professorship at NTU in 2006. 